Supporting Information

\title{
Development of a smart scaffold for sequential cancer chemotherapy and tissue engineering
}

\author{
Poulomi Sengupta ${ }^{\sharp, \S, \delta}$, Vinay Agrawal ${ }^{\phi}$, Bhagavatula L. V. Prasad ${ }^{\sharp, \S *}$ \\ \#Physical/Materials Chemistry Division, National Chemical Laboratory (CSIR-NCL) \\ Dr. Homi Bhabha Road, Pashan, Pune, 411008, INDIA \\ and \\ ${ }^{\S}$ Academy of Scientific and Innovative Research (AcSIR), Ghaziabad 201002, INDIA \\ ${ }^{\phi}$ Biopore Surgicals, Khar, Mumbai, 400052, INDIA
}

Ph: 91-20-25902013; Fax: 91-2025902636; Email: pl.bhagavatula@ncl.res.in

${ }^{8}$ Current address: Indrashil University, Rajpur, Kadi, 382740, INDIA 


\section{LIST OF FIGURES FOR SUPPORTING INFORMATION}

Figure S1. Calibration curve for the determination of unknown cisplatin concentration Figure S2. Scheme for cross-checking the role of citric acid's in conjugation with cisplatin Figure S3. UV-vis profile of AuNP-coated HDPE and cisplatin coated HDPE Figure S4.3D X-Ray Microtomography image of AuNP-coated porous HDPE scaffold Figure S5.Relative amount of platinum present in scaffolds before and after release experiments. Data acquired by a colorimetric assay using o-phenylenediamine hydrochloride. Data represented as the mean $\pm S D$ of $n=3$

Figure S6. Amount of platinum present in AuNP-coated HDPE scaffold and bare scaffold. Data acquired by colorimetric assay using o-phenylenediamine hydrochloride. Data represented as the mean $\pm S D$ of $n=3$.

Figure S7.Quantification of fluorescence from live dead assay (Figure. 5) using the Zeiss software Zen Pro 2012. Data represented as the mean $\pm S D$ of $n=3$.

Figure S8. Plotting of cells present in different quadrant for different treatments. (Data represented as the mean $\pm S D$ of $n=3$ ).

Figure S9.Expression of phosphatidyl serine on cellular membrane by imaging. Some representative images have only been presented here.

Figure S10. Quantification of fluorescence from each image in fibroblast regrowth experiment. The average fluorescence was obtained by measuring the fluorescence intensity with the software Zen Pro 2012 from $n=7$ areas.

Figure S11. Measurement of contact angle before and after drug release. Data represented as the mean $\pm S D$ of $n=3$.

Figure S11. Measurement of contact angle before and after drug release. Data represented as the mean $\pm S D$ of $n=3$. 


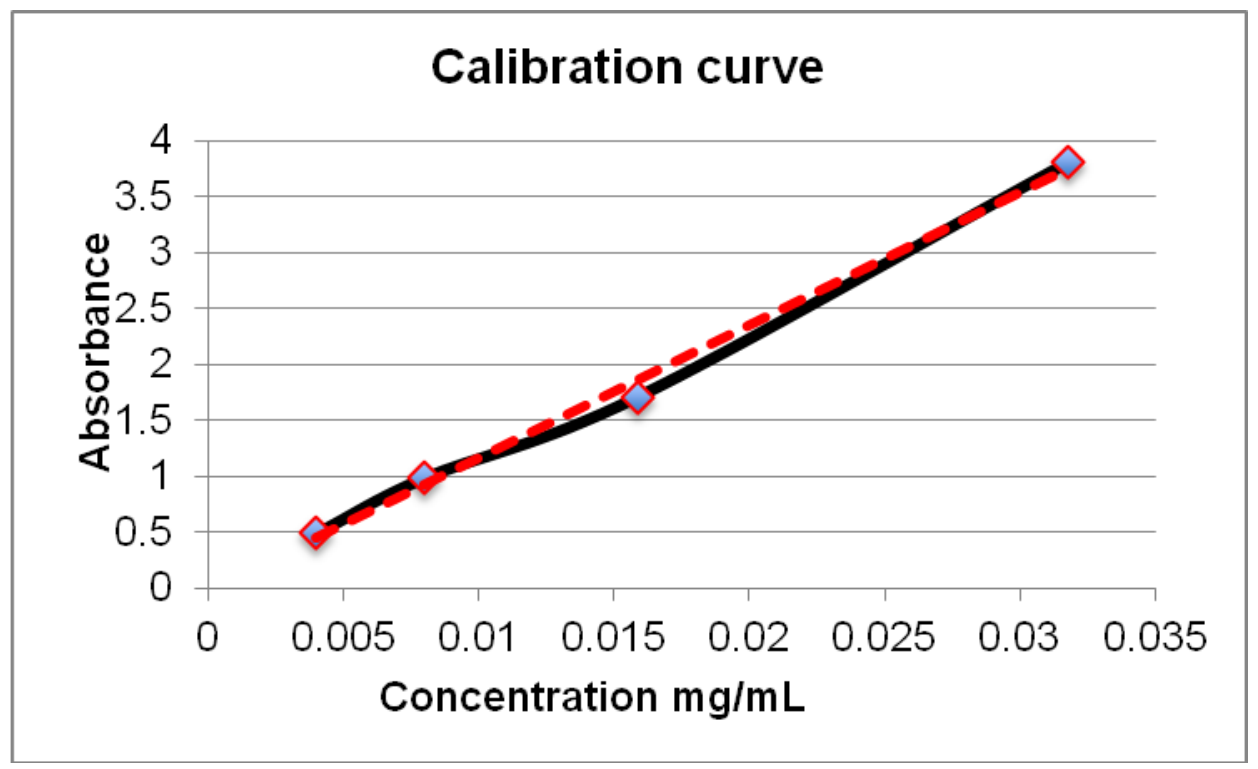

Figure S1. Calibration curve for the determination of unknown cisplatin concentration

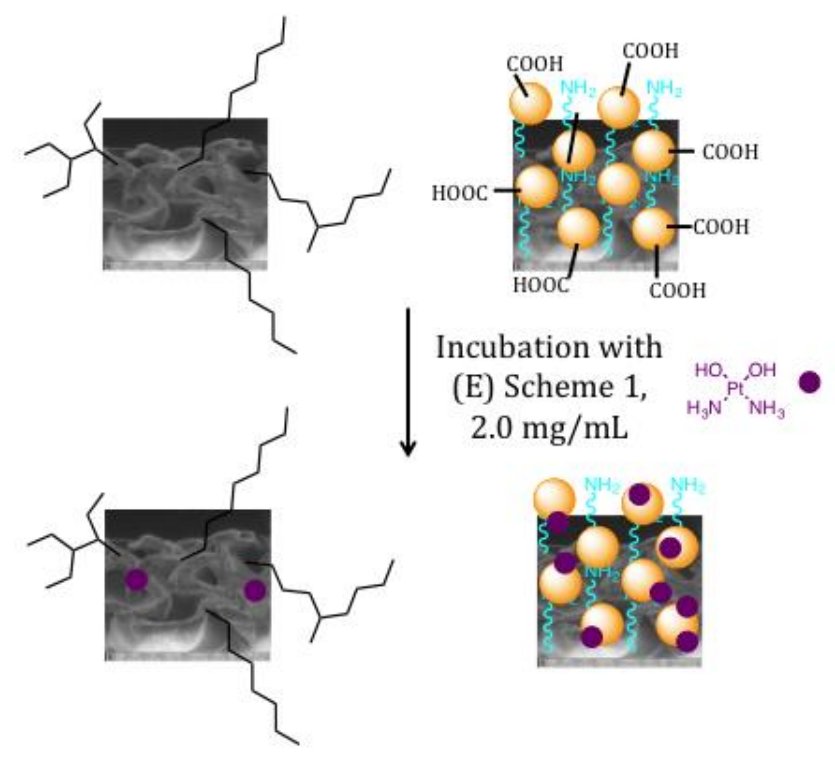

Figure S2. Scheme for cross-checking the role of citric acid's in conjugation with cisplatin 


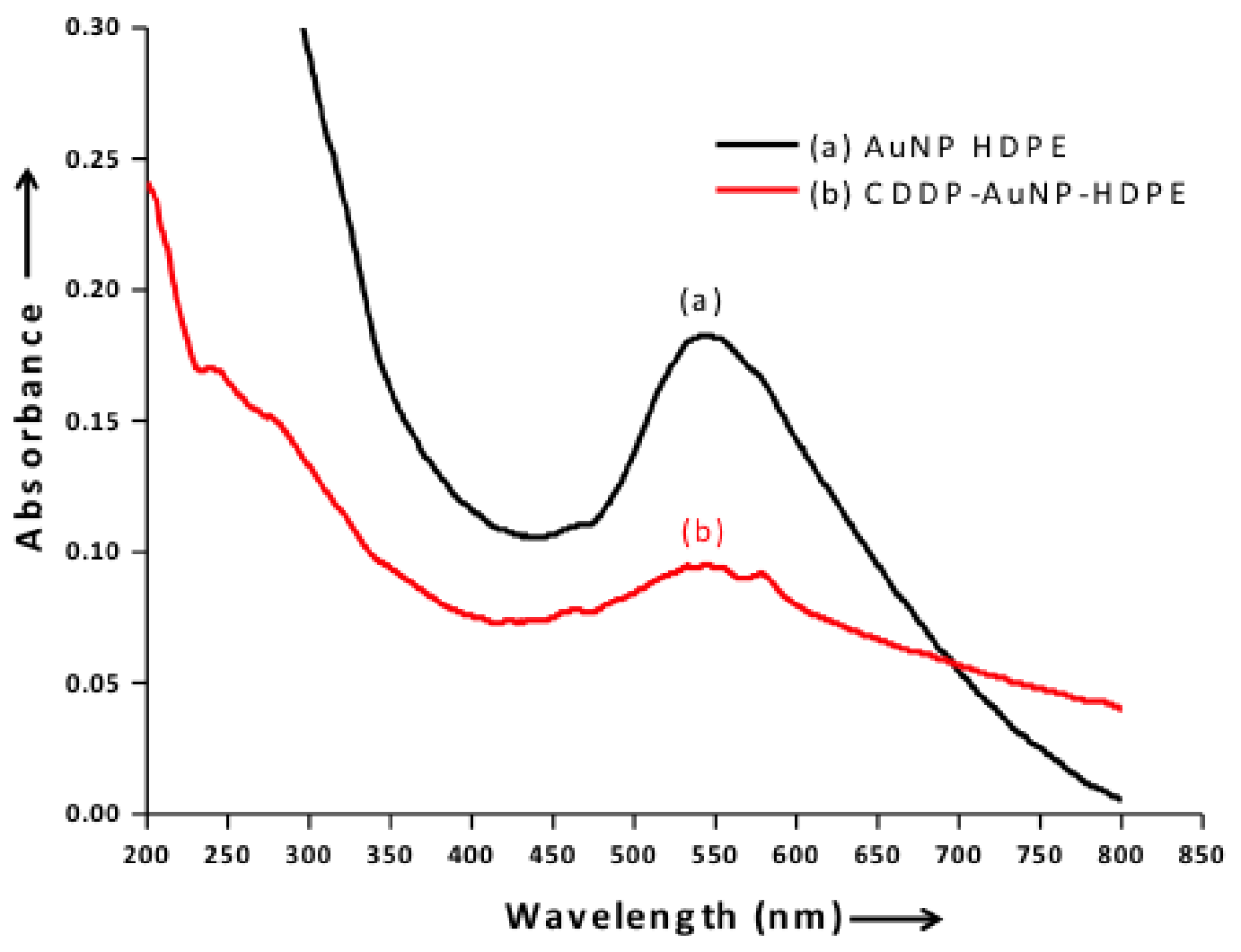

Figure S3. UV-vis profile of AuNP-coated HDPE and cisplatin coated HDPE 


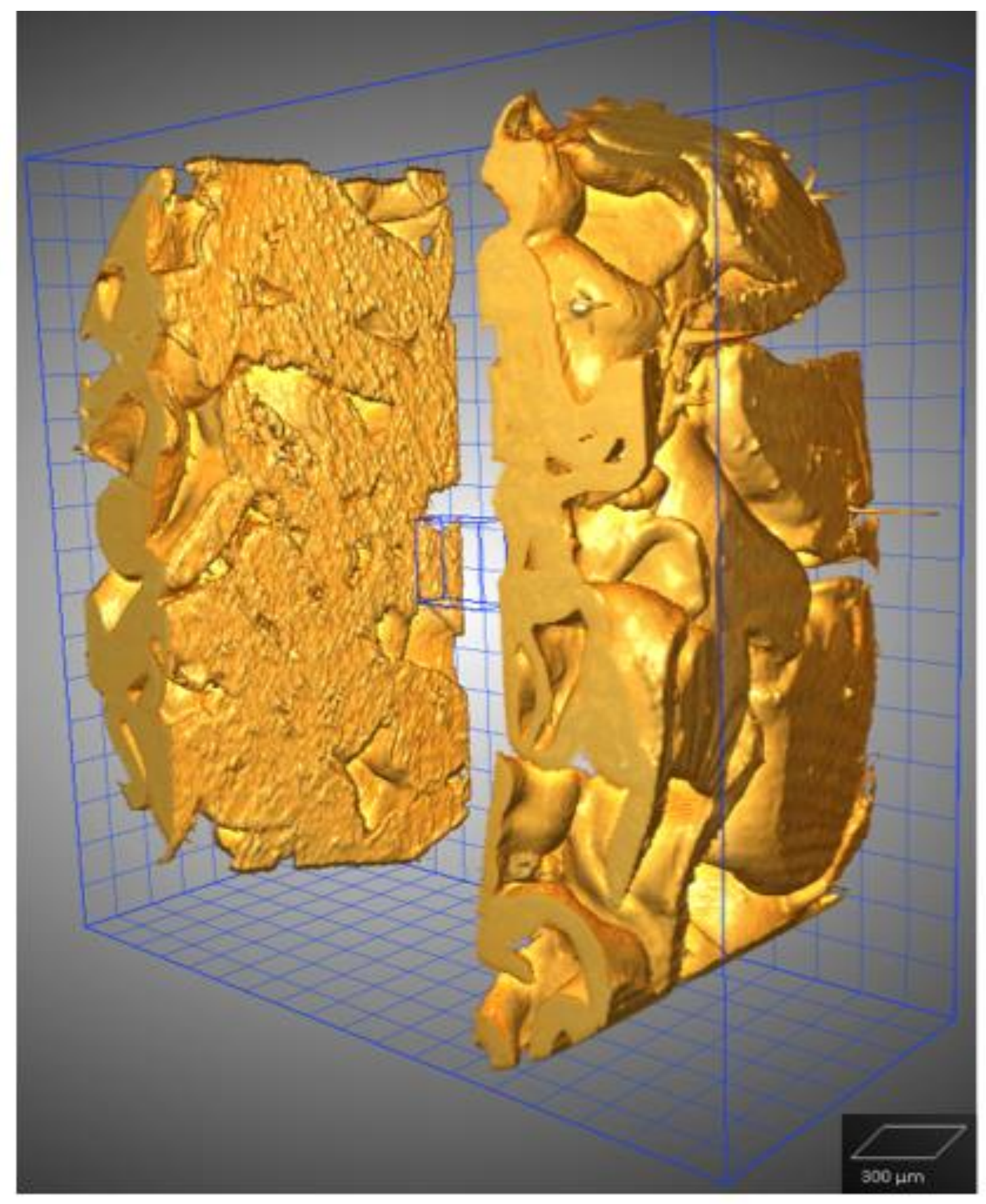

Figure S4. 3D X-ray microtomography image of AuNP-coated porous HDPE scaffold 


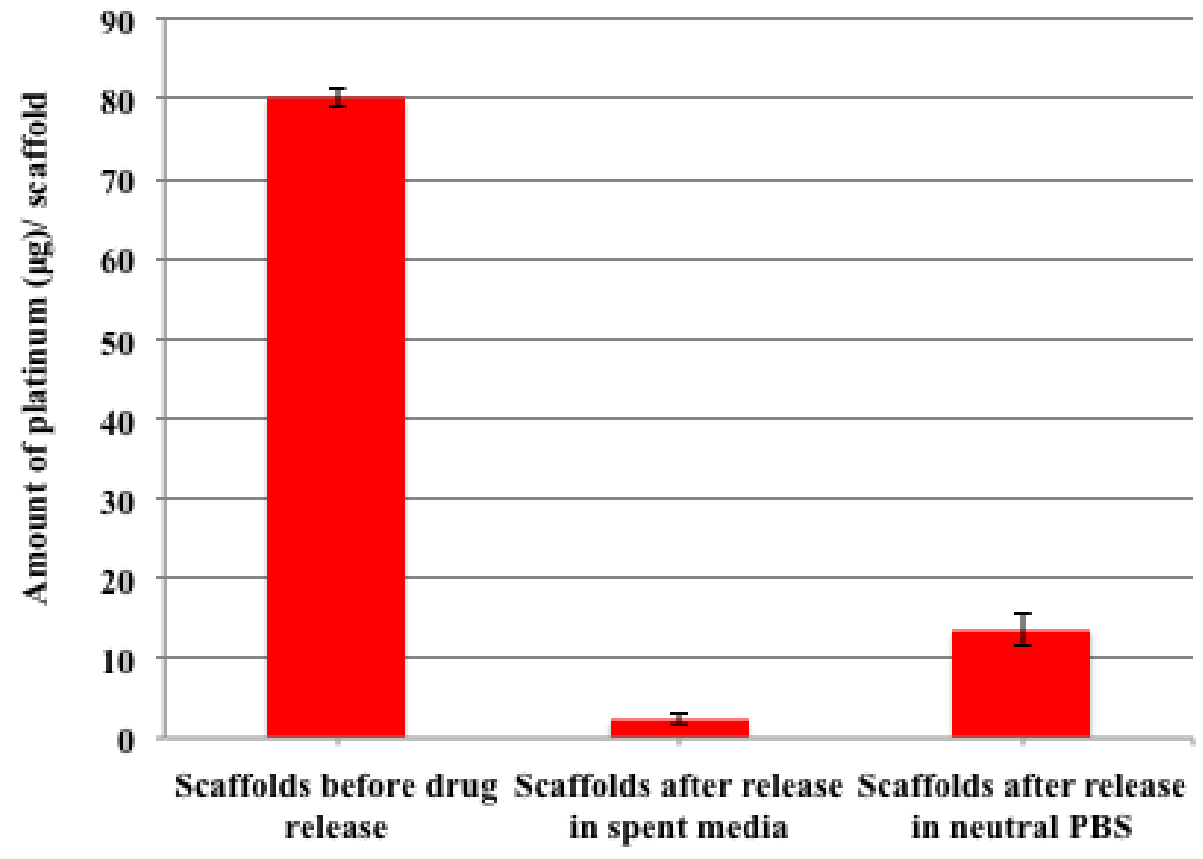

Figure S5. Relative amount of platinum present in scaffolds before and after release experiments. Data acquired by a colorimetric assay using o-phenylenediamine hydrochloride. Data represented as the mean $\pm S D$ of $n=3$ 


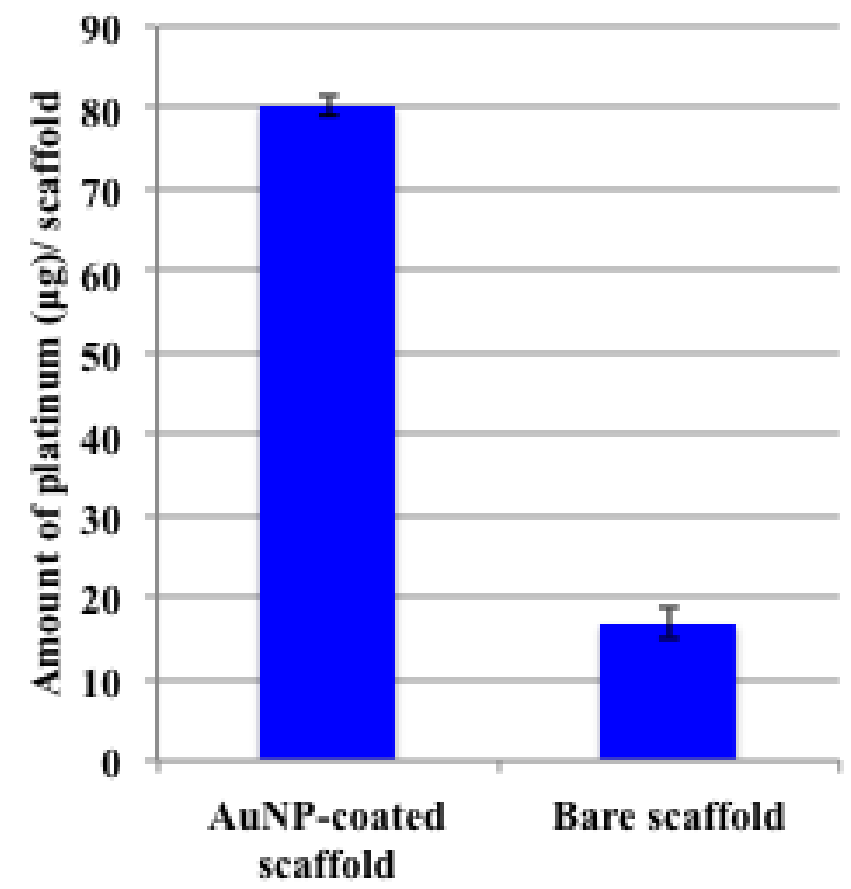

Figure S6. Amount of platinum present in AuNP-coated HDPE scaffold and bare scaffold. Data acquired by colorimetric assay using o-phenylenediamine hydrochloride. Data represented as the mean $\pm S D$ of $n=3$. 


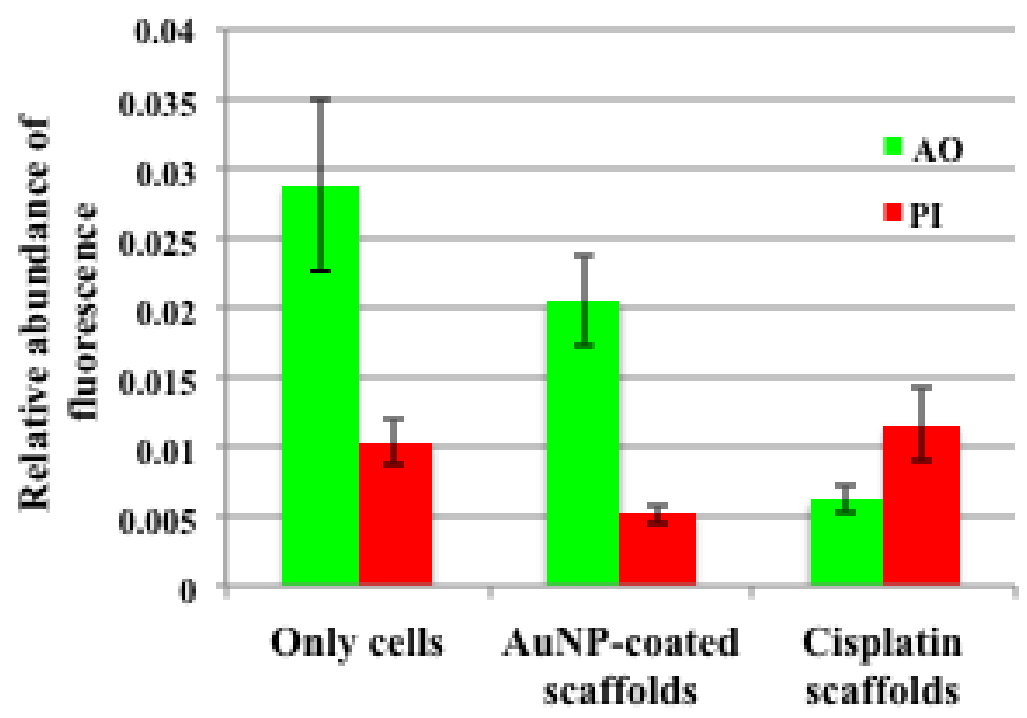

Figure S7. Quantification of fluorescence from live dead assay (Figure. 5) using the Zeiss software Zen Pro 2012. Data represented as the mean $\pm S D$ of $n=3$. 


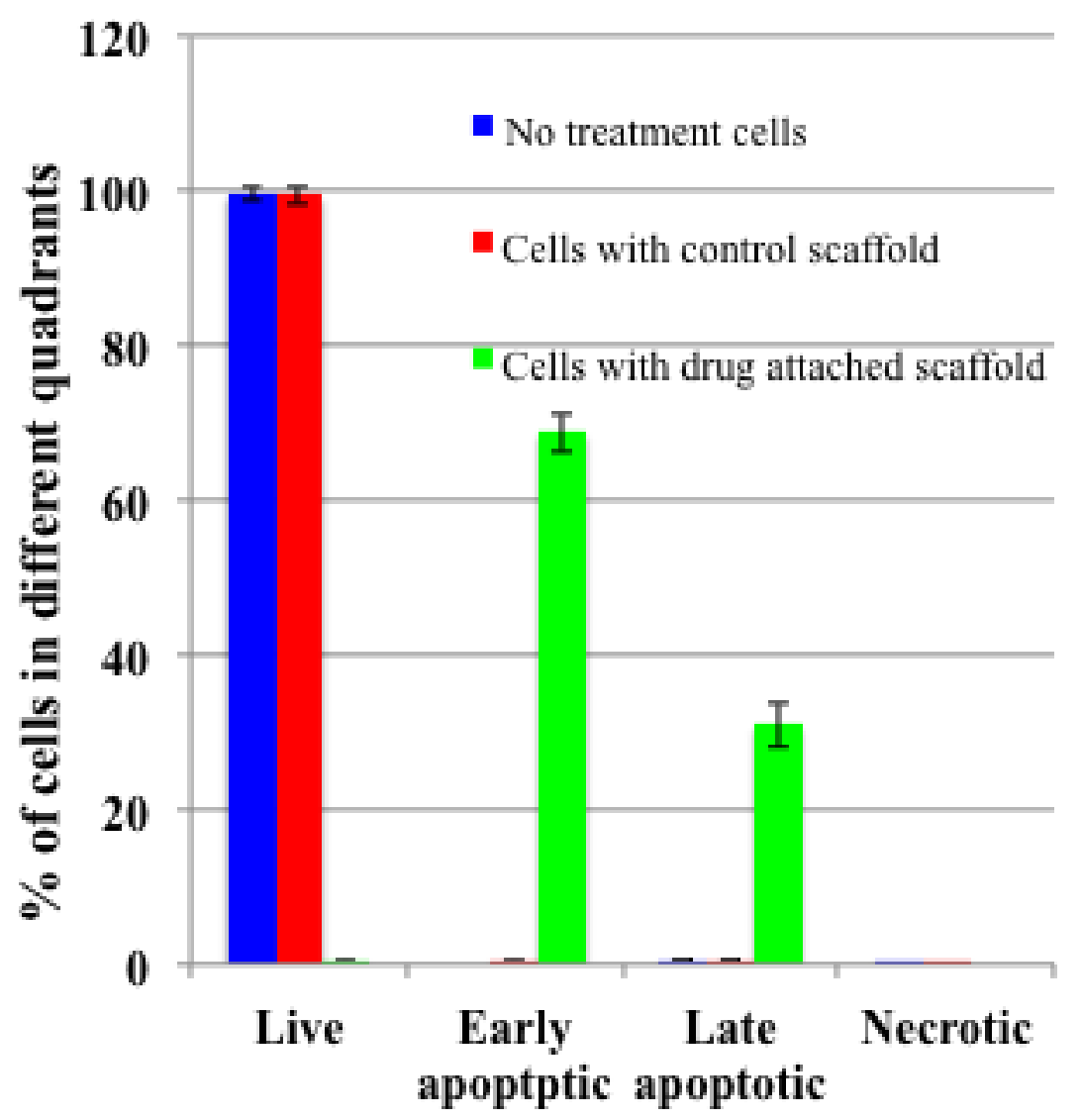

Figure S8. Plotting of cells present in different quadrant for different treatments. (Data represented as the mean $\pm S D$ of $n=3$ ). 

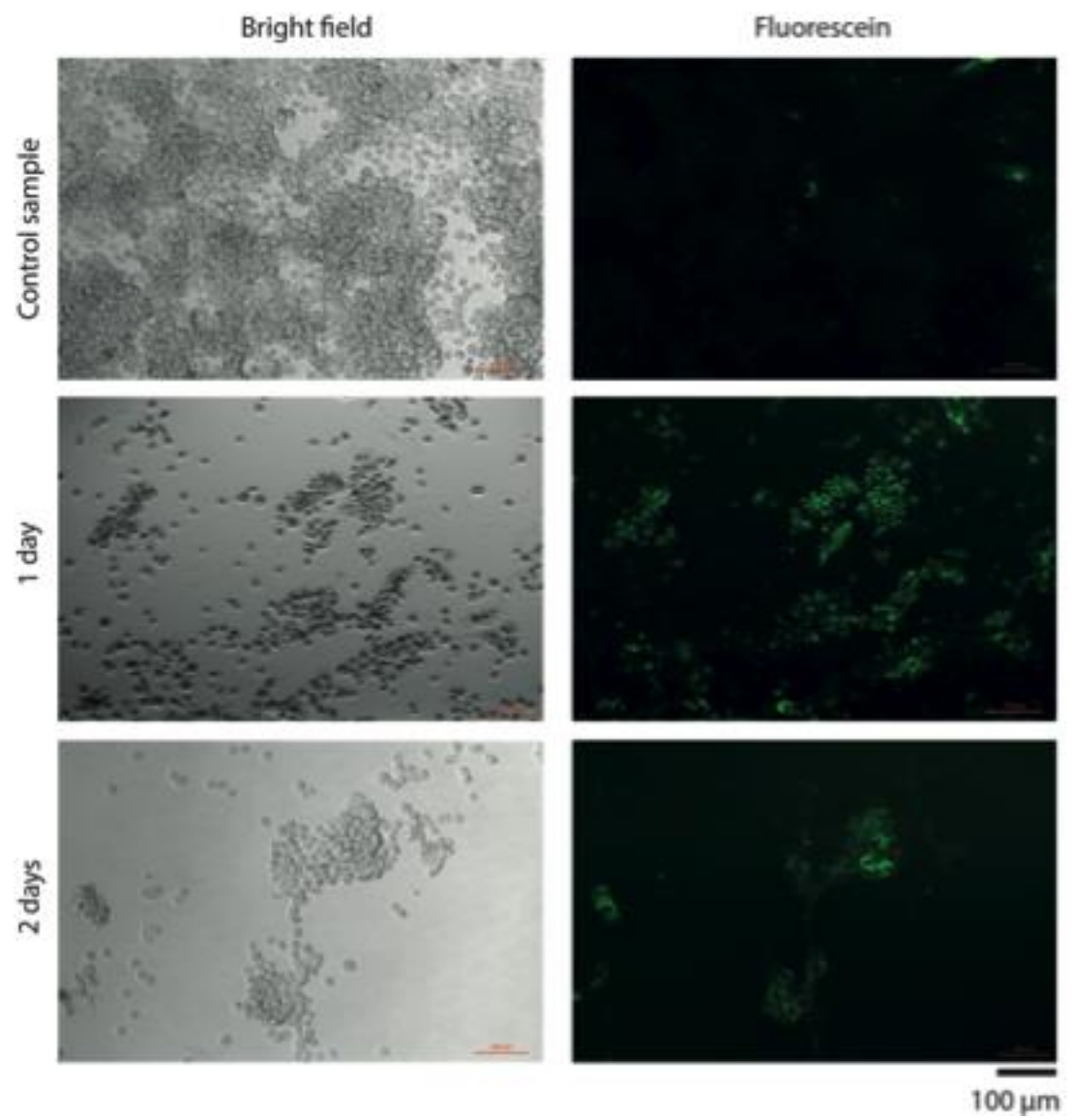

Figure S9. Expression of phosphatidyl serine on cellular membrane by imaging. Some representative images have only been presented here. 


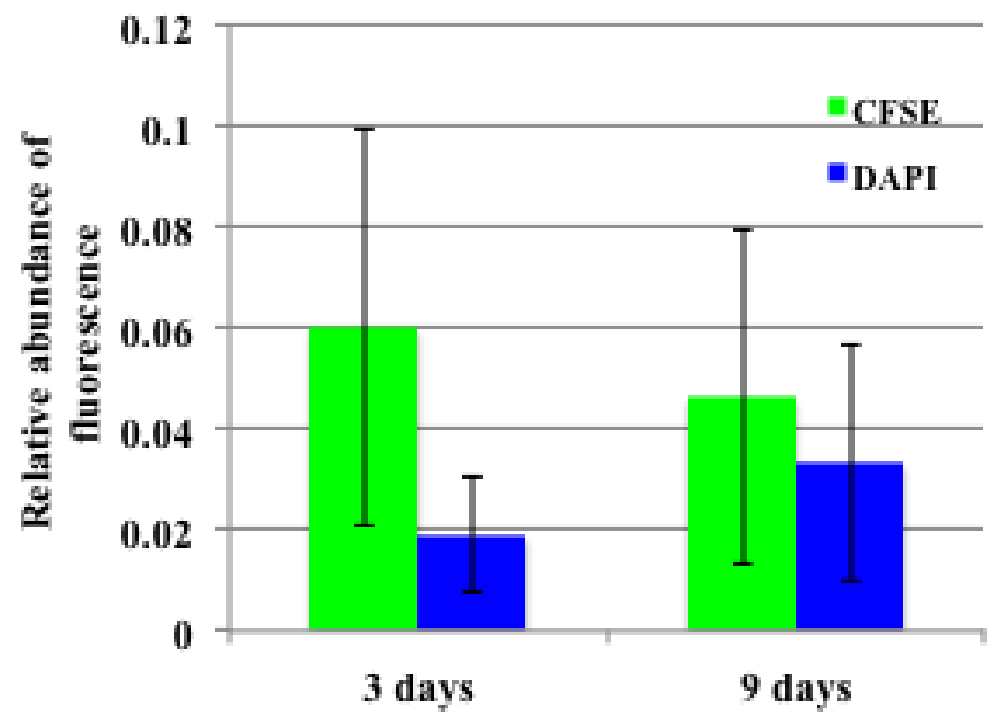

Figure S10. Quantification of fluorescence from each image in fibroblast regrowth experiment. The average fluorescence was obtained by measuring the fluorescence intensity with the software Zen Pro 2012 from $n=7$ areas. 


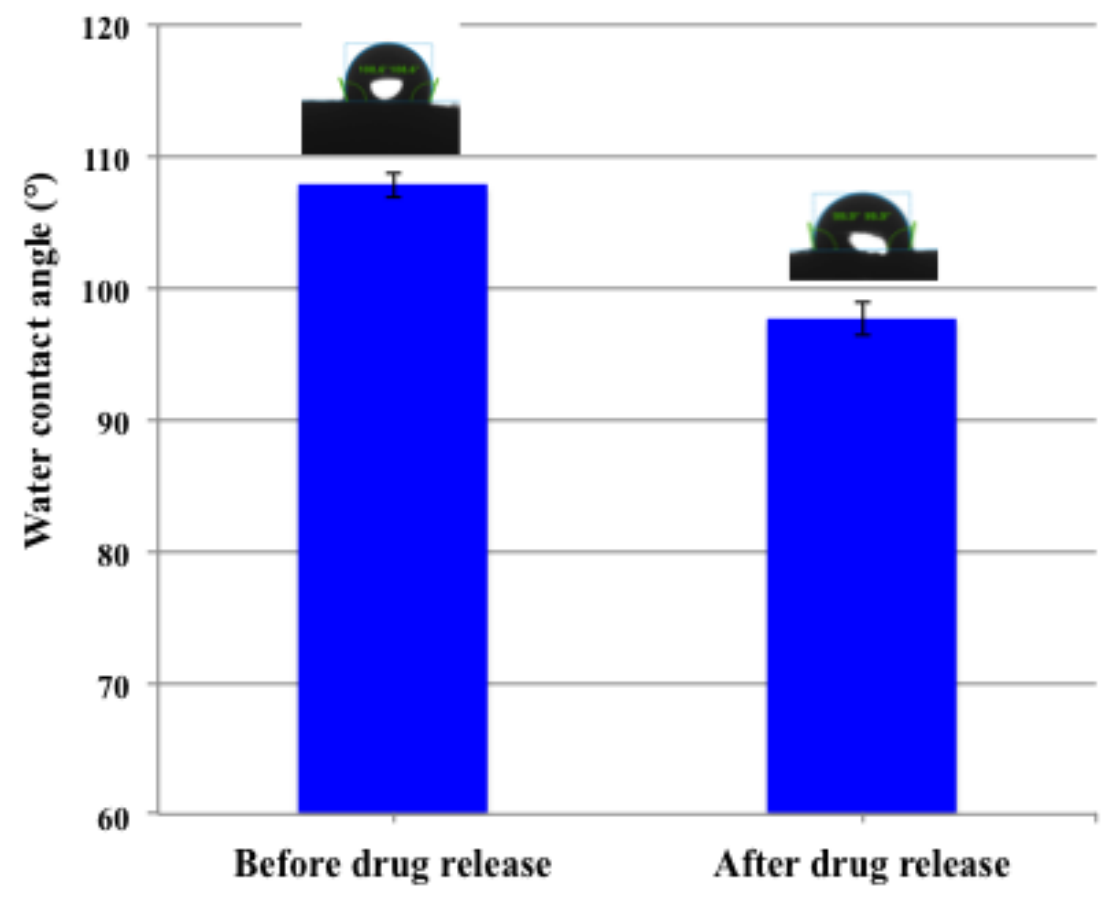

Figure S11. Measurement of contact angle before and after drug release. Data represented as the mean $\pm S D$ of $n=3$. 